\title{
ALGUNOS PROBLEMAS DE MÉTRICA GRIEGA: RITMO, PIES, METROS
}

\author{
M. Teresa Galaz
}

La métrica como parte del arte de la poesia es, por así decirlo, una

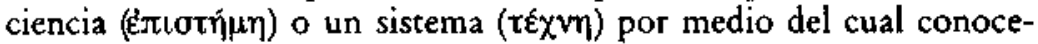
mos los principios de la versificación. Según Maas, en efecto, uno es el arte de la métrica, como medio de imponer un patrón regular al ritmo natural del lenguaje en una obra literaria, por lo que sugiere mejor el nombre de "ritmica" ", y otra distinta es la ciencia métrica, de la que afirma que «is the theory of the art of metric, and is therefore part of the study of poetry, as is, for example, the theory of poetic style. It is based on the phonetic values of the language, and is closely connected with the theory of rhythm in music and dancing ${ }^{2}$. En cuanto al carácter cognoscitivo, no creativo, de la métrica como ciencia, parecería entonces que no hay dudas. Éstas surgen, no obstante, en cuanto tenemos que determinar el campo donde se aplica este sistema métrico: porque si partimos de la estrecha relación que la poesía tenía con la música entre los griegos, y de que nuestra idea de ésta última se basa en una transcripción prosódica del texto poético -esto es, secuencia de sílabas breves (U) y largas (-) - en realidad no contamos con suficientes elementos para determinar cuál era la realización musical de los textos; en este sentido, podemos concordar con Del Grande en que dicha transcrip-

' Cf. de P. MaAs, Greek Metre, the Clarendon Press, Oxford 1962, p. 1.

2 Op, cit., p. 2. 
ción «se da un lato fornisce una linea metrica capace di appagare la nostra curiosità di indagine, dall'altro non sempre ci assicura che detta sequenza "metrica" abbia altesì pieno valore "ritmico" ".

Es por lo anterior, entonces, que han adquirido suma importancia los poquísimos textos en los que se ha logrado reconstruir la música y que, sin embargo, no aclaran el problema en un sentido definitivo ${ }^{4}$. De tal manera que, si bien se tiende a establecer una diferencia entre la métrica y la música ${ }^{5}$, hay estudios concretos sobre los fragmentos musicales que permiten aclarar algunos puntos dudosos en la primera ${ }^{6} \mathrm{y}$, sin duda, la confirmación o el pleno rechazo de algunas hipótesis dependerán de los nuevos descubrimientos que se hagan tanto en el campo de la poesía como en el de la música?. De cualquier modo, es posible para nosotros establecer que por lo menos hasta el siglo $\mathrm{v}$ la unidad métrico-musical estaba vigente ${ }^{8}$. Ahora bien, no es nuestro propósito hacer aquí una historia de los estudios sobre métrica a lo largo de la tradición filológica, ya que

3Véase C. Del. Grande, "La Metrica Grecan en Enciclopedia Classica, Sec. II, vol. 1, p. 152.

- Pese a que en realidad los textos con reconstrucción musical son pocos y están fragmentados, se encuentran obras de la importancia del Orestes de Esquilo (primer estásimo, vv. 338-343) o de la Pitica primera de Pindaro (wv. 1-5) para el siglo v. Hay también una ópera del ditirambógrafo Cinesias, de fines del siglo III, y un fragmento de monodia helenistica del siglo Il cuya melodia, empero, parece remontarse à la segunda mitad del siglo $v$; el resto de los textos, que oscilan entre el siglo i aC y la época cristiana (siglos III y IV dC), va desde peanes pertenecientes al Tesoro de Atenas en Delfos, hasta himnos que evidencian el sincretismo neopitagórico-cristiano en Egipto, pasando por el Epitafio de Sicilo, inscrito en una columna en Asia Menor. Véase, de C. Del Grande, op. cit., pp. 152-54 y 401-76.

s L. Gll, en F. Rodríguez, Adrados; M. Fernández-Galiano; L. Gil; J. S. Lasso de la Vega, Introducción a Homero, Guadarrama, Madrid, 1963, p. 193.

- Como la obra de E. Martin, Trois documentos de musique grecque, Paris 1953.

'En este sentido va la Greek Metre de SNELL aunque sin decirlo expresamente; tal es la razón de que Del Grande, que más bien por seguridad se inclina por la tradición, le acuse de whacer historia fuera de la historia* (Cf. id. p. 197).

${ }^{8}$ Cf. el fr. $1 \mathrm{D}, \mathrm{wv}, 6-8$ del tírico-trágico Pratinas de Friunte, donde se queja de que los instrumentos invadan al canto; asimismo, Dioniso de Halicarnaso, en De compositione verborum, 11, 64, donde afirma que en la poesía antigua la letra estaba sujeza al canto y no a la inversa. Podemos pensar, evidentemente, en la lírica monódica a la manera de Safo, donde todavía el cantor llevaba con el instrumento su propio acompañamiento, o inclusive en Esquijo y Sófocles que escribian sus acompañamientos; a partir del siglo $v$ se da la revolución musical que empieza con las improvisaciones de los flautistas (akletai), y que ocasiona q̨ue Euripides ya no escriba las partes musicales de sus obras. 
existen obras bastante especializadas acerca de ella"; tampoco intentamos reproducir ningún tratado sobre la materia, algo que sería inútil dada la cantidad de libros y artículos autorizados en el tema ${ }^{10}$. Así pues, nuestra intención es poner de relieve algunos puntos muy específicos de divergencia con la tradición que se han dado en la métrica funcionalmente, siempre en cuanto a la relación texto-ritmo.

Sabemos, en efecto, que los griegos tenian tres formas de ejecución

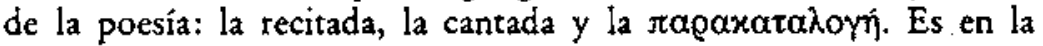
primera forma que se interpretan el hexámetro dactílico, el trímetro yámbico y el tetrámetro trocaico, si bien en una prehistoria épica el hexámetro había sido cantado. En la segunda forma, el canto, se interpretaron la monodia y la elegía, entre otras, y es la misma palabra ழپón la que, al parecer, indica la innovación del canto y

9 Se puede ver la pequeña relación de P. MaAs en su Greek Metre, ya citado, esp. pp. 1-8, así como el prefacio del Manual de prosodia y métrica griega de M. Lenchantin de Gubernatis, UNAM, México 1982, pp. 7-16; pero, indudablemente, para tener una verdadera perspectiva de lo que ha constituido la tradición métrica desde el siglo XIII, en que resurgió, hasta $\mathrm{J}_{a}$ actualidad, es indispensable consultar aLa Metrica greca*, de C. Del. GraNDE, esp. las pp. 155-213. En cuanto a las fuentes, tenemos los principales elementos de argumentación en los fragmentos de los estudiosos de la antigüedad, como Damón y Arquitas de Tarento (ed. de Diels); Platón (Rep. III, 10-12, 398 c-403 c); Aristóteles (Poética; Retórica; Politica, tib. VIII y los Problemas musicales, definidos como inciertos por algunos editores pero incluidos en el corpus); y su discípulo Aristóxeno de Tarento (Elementos rímicos, varias eds.). Estudios más sistemáticos de la época helenística, a la vez que mejor

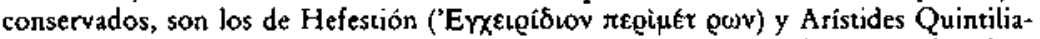

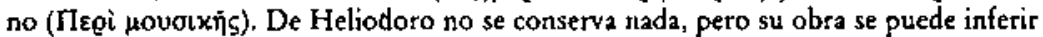
de los escolia a Aristófanes (Cf. de ]. W. WHrt:, The Verse of Greek Comedy, Macmiltan, Londres, 1912, pp. 397-421); asimismo tenemos el tratado De compositione verborwm de Dioniso de Halicarnaso y el Пepi novouxn̂s de Pseudo-Plutarco. La brevísima introducción de Snell, por úttimo, nos pone al tanto del estado de la cuestión (Cf. del atutor ta Metrica greca, trad. y ed. La Nuova Italia, Florencia 1977 , esp. Pp. 1-2).

10 Se puede ver, entre otros, los ya citados de Del Grande y Lechantin, quienes se inclinan más por seguir la tradición, igual que Fesra (Richerche metriche. Saggio di kn nuovo metodo per lo studio della metrica greca, Sandron, Roma 1926) que además se pronuncia claramente en contra de los ritmicos. El tnanual de B. GeN\}ILI, La metrica dei greci (D'Anna, Florencia 1955), no tan conservador y si muy justrativo, contrasta con la obra de Snell del mismo año, por cuyo contenido éste último de ninguna manera puede ser catalogado como simple manual, sino más bien como un opúsculo teórico a la manera del de Maas. Sobre las tendencias modernas sincrónicoestructurales en métrica se puede consultar, de M. SANCHEZ RUIPEREZ, "Ideas fundamentales sobre métrica griegan, en Estudios Clásicos, I 1950-52, pp. 239-55. 
excluye la recitación ${ }^{11}$. La $\pi \alpha \rho \alpha x \alpha \tau \alpha \lambda o \gamma \eta$, en cambio, era la forma mixta de una recitación con acompañamiento musical; de ella dice el

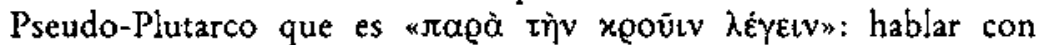
énfasis, pero sin cantar, $y$ atribuye su invención a Arquíloco ${ }^{12}$. En cualquiera de las tres formas, el ritmo se presenta como un mecanismo basado en la repetición que da cadencia a los diversos grupos melódicos; en la lengua griega de la antigüedad había un sistema rítmico muy diverso al de las lenguas actuales, esto es, era un sistema de naturaleza "cuantitativa», a diferencia del sistema "dinámico", según llama Maas al metro bizantino y, por extensión, al ritmo de las lenguas modernas ${ }^{13}$.

Por lo que respecta al elemento principal de diferenciación entre ambos sistemas, "cuantitativo" y "dinámico", éste se presenta en relación al modo de acentuación (ictus), que en el primero consiste en un cambio de tono (acento melódico) mientras, en el segundo, en la enfatización de un miembro de frase con respecto a los demás, sin que se dé ninguna variación total (acento enfático). De aquí que se haya intentado equiparar la secuencia sílaba larga (-)-breve (U) con la secuencia sílaba tónica-átona, y que se haya persistido en la utilización de una terminología como la de "arsis" y "tesis", que ya Maas consideraba poco apropiada ${ }^{14}$. Efectivamente, dichos términos evocan más bien el énfasis o la falta de ese énfasis en las lenguas modernas, mientras que en el griego antiguo la distinción de sílabas se da en razón a su cantidad, fenómeno que en un idioma como el nuestro nos deja completamente indiferentes. No obstante, en las palabras "arsis" y "tesis", que musicólogos antiguos como Baquio y Querobosco utilizaban a la inversa, y que fueron confundidas desde Atilio Fortunaciano ya en época romana, tampoco se encontraba el sentido de "larga» y "breve» que Maas atestigua para sus contemporáneos, sino que se referían más bien al momento en que se golpeaba con el pie, marcando el compás, o se batian las palmas (tesis) y al momento en que esa acción se suspendía (arsis). De aquí la hipótesis que sostiene la ausencia de un ictus dinámico ante un sistema de composición basado en el ritmo cuantitativo sin ictus, ritmo que, por otra parte, ha sido comprobado en investigaciones realizadas entre

"Cf. de F. PERusino, Il tetrametro giambito catalettico nella commedia greca, Ed. dell'Ateneo, Roma 1968, p. 22.

12 Véase, del autor, De mus., esp. 28, 1141 a-b.

${ }^{13}$ Cf. op. cit., pp. 1 i 3.

14 Ibid, p. 6. 
grupos eslavos que permanecieron aislados, por lo que pueden contemplarse como los sucesores del antiguo sistema cuantitativo griego, entre quienes no hay reminiscencias de un sistema dinámico ${ }^{15}$.

Ahora bien, Maas nos propone, en vez de la utilización de los conceptos de "arsis" y utesis" que privaban entre sus contemporáneos y, en general, en cualquier autor que se declare seguidor de la tradición ${ }^{16}$, su doctrina de la "responsión» interna y externa; esto es, entre miembros de un mismo verso y entre versos de una estrofa, o aun entre varias estrofas. Con el objeto de apoyar su teoría, Maas encuentra los elementos ubiceps" (은) que en general se fundirán luego con los "anceps" ( $x$ ). A partir de la responsión hemos podido resolver fenómenos como el de ubrevis in longon, como por ejemplo en el siguiente verso de Arquíloco ( $\mathrm{fr} .74 \mathrm{D}$ ), que por responsión interna requiere de una sílaba larga para el segundo troqueo cataléctico:

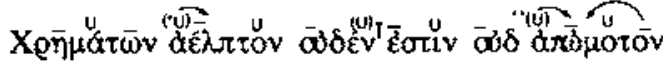

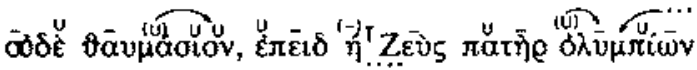

Esquema: 2 tro 2 tro $=4$ tro

+ Zeugma de Havet

++ Zeugma de Wilamowitz-Knox

+++ Zeugma de Hermann para el tetrámetro trocaico

++++ Corte de palabra obligatorio ante cesura medial

is Véase, de T. Georgeades, Der Griegische Rhytmus. Musik-Reigen, Vers und Sprache, M. von Schroeder, Hamburgo 1949. Esa hipótesis ha sido desarrollada extendiéndola hasta el verso latino; se puede recordar aqui que éste no estaba totalmente ajejado de la música, como nos hace saber la mención virgiliana en la Bucólica V, vv. 13-15, de una cierta notación musical en la corteza de un árbol, que hace pensar en un primitivo pentagrama; acerca del kexperiar», se hace hincapié en que *las canciones requerian ensayo* (Cf. de J. A. MARTiNEZ CONESA, sSobre métrica griega», Estudios Clásicos, 64, 1971, p. 370). Cf. A.W. DE GROOT, *La métrique génerale et le rhytmen, Butlecin de la Saciété de linguistique de Paris, XXX, 1930, pP. 202-32, y posteriormente *Der Rhytmus*, en Neophilologus, XVII, 1932.

16 Confróntese el argunento de los «métricos» en M. LENCHANTiN, op. cit., p. 9: - El acento griego era prevalentemente, no únicamente, melódico. Ahora está probado que no se da aumento de tono sin aumento correlativo de intensidad dinámica. La opinión de que la alternancia de silabas de distinta cantidad, reunidas en torno a la cima tónica representada por el acento, produjese una melodía rudimentaria, transformaría en melopeya toda la lengua griega, no sólo en las obras de poesia, sino también. en la conversación ordinaria: cosa sencillamente absurda." 
o el de un hiato que no abrevie ante pausa, como en los siguientes versos de Sófocles (Oedip. Rex, 1373-75):

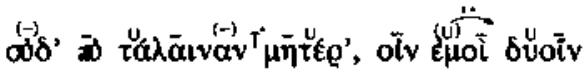

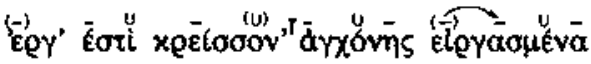

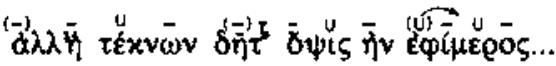

Esquema : $=3$ iall

+ Cesura después del segundo anceps, aunque no es obligatoria en los yambos

++ Zeugma de Porson

$o$, en fin, del encabalgamiento (enjambement), en el que una frase que habitualmente terminaría con el verso, por el sentido sigue con el término siguiente ${ }^{17}$; aunque no es una figura característica de la épica, lo encontramos, sin embargo, hasta en tres ocasiones en un pequeño grupo de versos de la lliada (37 al 52):

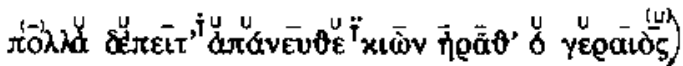

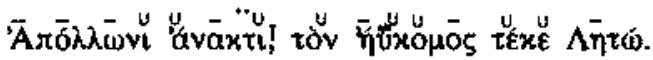

$$
\begin{aligned}
& \text { (vv. 35-36) }
\end{aligned}
$$

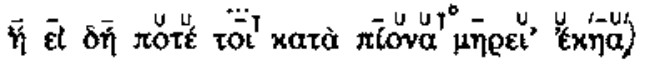

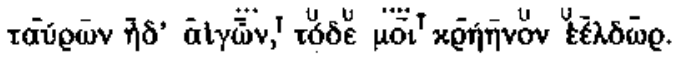

$$
\begin{aligned}
& \text { (vv. 40-41) }
\end{aligned}
$$

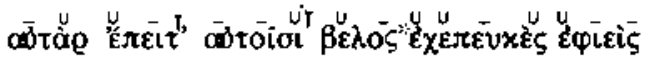

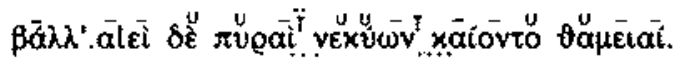

$$
\begin{aligned}
& \text { (vv. 51-52) }
\end{aligned}
$$

${ }^{7}$ Cf. de DAIN, Leçan sur la métrique grecque, Klincksiek, Paris 1944, donde desecha la teoría de los pies y propone una medición sobre *elementos métricosn, con lo que explica el enjambement; este último fenómeno, por otra parte, aparece en el hexámetro por lo general ante epitetos y está considerado como una suerte de

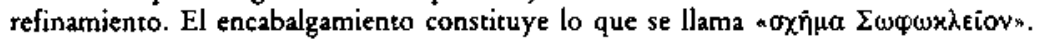


* Homero utiliza el arcaísmo sin aspiración para lograr la segunda breve del

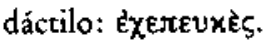

+ Cesura triemimera

++ Cesura trocaica

+++ Cesura pentemímera

++++ Cesura heptemímera

b Diéresis bucólica

Por lo que se refiere a la cantidad, fue Hermann el primero en

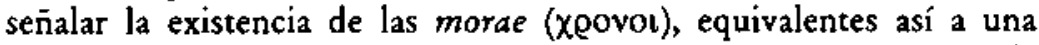
unidad de tiempo como a una de espacio, en una secuencia de tiempos y espacios alternantes sobre la que operaba el ritmo. Esto es importante porque en 1945 un experimento permitió comprobar que una vocal larga no sólo no ocupaba dos veces la cantidad de tiempo de una breve, sino que había largas que cronométricamente duraban menos tiempo que las breves ${ }^{18}$. Así pues, se llegó a la conclusión de que la cantidad vocálica no era el equivalente a la duración cronométrica, y que la cantidad en las vocales griegas se debía medir funcionalmente, es decir, según el papel que estuvieran jugando en la frase. Es explicable entonces por qué cuando se habla del dáctilo (一UU) se dice que kil longum e i due brevia sono equivalenti nel senso che al posto de due brevi può stare quasi sempre anche una lunga, ma il longum non può essere rapresentato da due brevi ${ }^{19}$." De aquí que Mas defina a la seguna mitad dactílica como "biceps" $(\stackrel{\cup}{-}$ ), mientras que Snell encuentra un dáctilo trisiläbico ( $-U U$ ) o bisilábico ( - ).

Al principio de responsión también se debe, por otra parte, un replanteamiento en la organización de los elementos métricos, que tradicionalmente se agrupaban en pies y metros. De los primeros

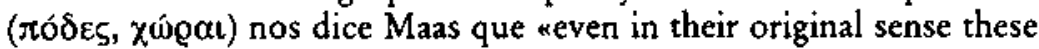
terms are useless, because they make a unit not of the organic groups of elements but of parts of these arbitrarily separated from the wholes ${ }^{20} . "$ Asimismo, sabemos que el concepto de "pie" como unidad mínima de medición proviene de los métricos posteriores, como Hefestión (siglo II aC), ya que en una tradición más antigua encontramos referencias sólo a «ritmos»; es así, en efecto, cuando

18 En efecto, en una tesis de ese año, M. Durand hace estadjisticas sobre la duración cronométrica de las vocales y encuentra que, en términos generales, las breves duran entre 5 y 27 centésimas de segundo, mientras las largas duran entre 15 y 36 .

19 Véase, de B. SNELt, Metrica greca, op. cit., p. 11.

2o Op. cit., p. 7 . 


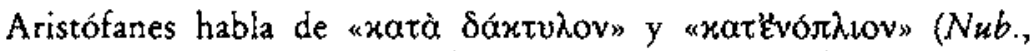
651), que indicaban series de versos y no pies aislados. A este respecto Gentili comenta que aè certamente un errore usare ancora termini come pentapodia giambica, trocaica, dattilica, perchè la più piccola unità metrica non è il piede giambico, trocaico, dattilico, ma il metron o dipodia giambica, trocaica, dattilica che rappresenterano unità metriche indivisibili ${ }^{21}$." Encontramos entonces que los versos se agrupan por unidades métricas o metra, y por unidades rítmicas o cola; de hecho, los cola no son otra cosa que la apariencia visual que los alejandrinos dieron a los versos con el objeto de editarlos, ya que hasta el siglo Iv todo texto era escrito "en prosa", es decir, sin separación de líneas. Los cola son, pues, los versos por línea cuando se da responsión externa en una estrofa, y sólo aparecen en la poesía cantada; para Maas el término colon también es válido en aquellos casos en que varios grupos de metros respondan internamente como un todo ante otros grupos similares ${ }^{22}$.

Por último, la estrofa, que consta de unos seis u ocho metros, puede subdividirse a su vez en períodos que se separan entre si por medio de pausas, mientras, en correspondencia, las unidades métricas se separan por medio de cesuras y diéresis, en general marcadas en lugares fijos que no estropean el buen sentido de la frase, sino que en general lo siguen, como podemos apreciar a partir de los siguientes disticos de Arquíloco (fr. $6 \mathrm{D}$ ):

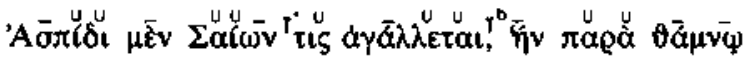

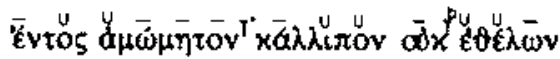

En efecto, si bien en los dos pentámetros se cumple la cesura medial (pentemímera o "masculina"), con final de palabra obligatorio, en los hexámetros se da la misma cesura y además la diéresis bucólica coincidiendo con final de palabra, que facilita el sentido.

No intentaremos aquí reproducir las variantes de metros o de estrofas que se encuentran ilustradas con profusión en los

21 Cf., del autor, La metrica dei greci, op. cit., p. 2. En los mismos têrminos está la nota aclaratoria de B. SNE.L. (op. cit., p. 3), para quien $x$ giambi, trochei e anapesti compaiono però solamente come "metri" e chiamare questi ultimi "doppi piedi" (dipodie) non fa che creare confusione*.

22 lbid., p. 38 . 
manuales ${ }^{23}$; baste señalar que, al contrario de la tendencia de la tradición a fijarlos y esquematizarlos, en la métrica postmaasiana se ha reconocido la libertad de que tales formas gozan, incluyendo el hexámetro homérico, en el que ya Hefestión habia señalado las únicas treinta y dos posibilidades, comprobadas después en la práctica ${ }^{24}$. Pero si el mismo hexámetro homérico era en extremo libre, contrastándolo con el de la época helenística, se puede hablar todavía de mayor libertad entre los metros pertenecientes a la poesia "cantada", ya fuesen dáctilos, yambos, troqueos o anapestos. Así, los dáctilos se presentan en trimetros (Estesícoro), tetrámetros (Ibico), hexámetros, octómetros (Alcmán) o heptámetros (Arquíloco); con preferencia hacia los dáctilos puros en lugar de los espondaicos, pero con la libertad de colocar una sílaba larga (-) o dos breves (UU) antes de un periodo, lo que le da una dirección "ascendente", como se puede apreciar en el siguiente ejemplo de Ibico (fr. 7 D):

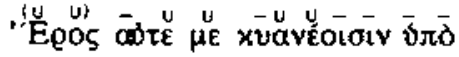

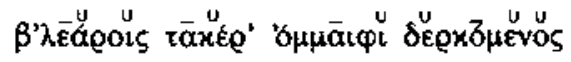

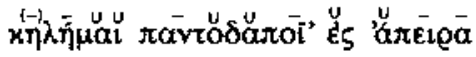

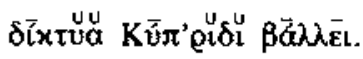

Esquema:

$$
\begin{array}{r}
U \cup 8 d a \\
-\quad 4 d a \\
3 d a
\end{array}
$$

o inclusive una sola mora breve (U), como en el siguiente fragmento de Estesícoro $(15,1 \mathrm{D})$ :

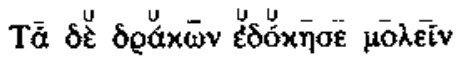

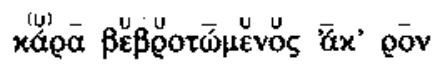

Esquema:

$$
\begin{array}{r}
4 \text { da } \\
\cup 3 \text { dal }
\end{array}
$$

${ }^{23}$ Nos referimos a los ya citados de Lenchantin y Gentili, o a la obra misma de Maas.

${ }^{24}$ Cf. el artículo *Glosas al hexánetro homéricon, de J. A. Martinez CONESA, Estudios Clásicos 64, 1971, esp. pp. 378-82. 
Asimismo, los versos gozan de gran libertad en el final del metro, cosa que difícilmente se da en los versos recitados, pese a los ejemplos del tetrámetro yámbico cataléctico $(x-U-|x-U-| x-$ $\mathrm{U}-|\mathrm{UU}-=2 \mathrm{i} a| 2 \mathrm{ia})$ y del trímetro yámbico cataléctico o escazonte $(x-U-|x-U-| U-=3 i a \wedge||)$. Los dáctilos, por ejemplo, se presentan catalécticos, con varias posibilidades:

$$
\begin{aligned}
& -\cup U-\|(2 \mathrm{da} \wedge \hat{\wedge} \mid) \\
& -\cup \cup-U-\|(2 \mathrm{da} \cup-\|) \\
& -\cup \cup-U--\|\left(3 \mathrm{da} \cup{ }_{\wedge}^{-}\right.
\end{aligned}
$$

al igual que los anapestos, que además presentan variantes de largas por breves, llegando a darse muchas posibilidades de substitución en formas como la siguiente: $\overline{U U} \cup U$ UU UU | UU UU UU - | $\overline{U U}$ UU UU - UU - - $\|$. Lo anterior ha dado materia para hablar de elementos yámbicos o trocaicos en los finales, o ha ocasionado polémicas en relación a si se trata de ritmo crético $\left(-U_{-}\right)$o de peónico, lo que complica aún más la cuestión porque el peón (-U一) es susceptible de resolverse de otro modo (-UUU) ${ }^{25}$. Es posible, inclusive, que la base eólica que se da en los líricos ( $\mathrm{xx}$ ) pueda aparecer acéfala (monosilábica) como en la variante llamada telesileo para un gliconio ( $-\cup-\cup \cup-U-)$, metro ya de por si problemático en la interpretación de las estrofas alcaica o sáfica: $\mathrm{x}$-UU-U- ( $\wedge \mathrm{gl}$.).

Tales elementos, añadidos o eliminados al principio o al final de los metros, en ocasiones sólo se explican por la libertad de responsión ${ }^{26}$, y es un hecho que aun un autor esquemático como Festa debe dar a sus cola "primordiales" cierta fluctuación para que en un momento dado sirvan para el análisis de un verso; fluctuación que, por otro lado, va desde el "pie" o la "dipodia" hasta elementos tripódicos y tetrapódicos, a los que se suman a veces algunos otros; de modo que los "metros primordiales" son resueltos por el autor en ocasiones como "asclepio + adonio", "hexámetro con inicio coriámbico y final báquico», y una sigla « $\mathrm{H}^{1}$ » que se refiere a «una tenue

25 Respecto a estos dos metros en series yámbicas y trocaicas "sincopadas", véase de J. A. Martinez CONESA, "Sobre métrica gtiegan, op. cit., esp. pp. 372-75.

${ }_{26}$ T2l es la posición de B. SNELL, por ejemplo, en op. cit., p. 28 , donde se refiere concretamente al dáctilo. 
corriente del lekythion que se expande con muchos dáctilos y anapestos hasta $[\ldots]$ las vibraciones yámbicas finales ${ }^{27}$ ".

En el presente trabajo hemos intentado presentar algunos puntos, dentro de la teoría métrica de la lengua griega, que se han presentado como problemas insolubles en la tradición filológica; hemos referido, por ello, posiciones situadas precisamente frente a esa tradición que, aún sin dar soluciones definitivas, modifican cada vez más las premisas. Es evidente que, en todo caso, los descubrimientos en poesía y en música son los que habrán de determinar las nuevas corrientes. Para finalizar, no obstante, nos parece oportuno mencionar una importante observación de Maas en relación a que «the freer metrical treatment of the trimeter in comedy was made necessary, and also offset, by the observance of stricter grammatical and prosodical rules (e.g. the purity of the dialect, the preference of xugí $\alpha \lambda \xi \xi \zeta$, the use of the article, the treatment of vowels preceeding a mute and a liquid, ec. $)^{28}$. Esto es, en otras palabras, que inclusive esta libertad de responsión estaba medida de acuerdo a la tradición gramatical y no se daba arbitrariamente, a la vez que cada metro tenía funciones específicas; por ejemplo, en la tragedia y la comedia posteriores, las variaciones en el metro servían para marcar efectos de movimiento rápido, en la primera, o de comicidad en la segunda, y tal parece que el tooxaĩos (troqueo) daba, en efecto,

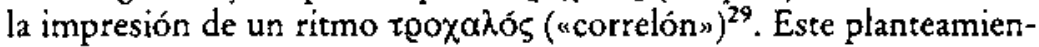
to maasiano se ha llevado a efecto, en los últimos tiempos, en un interesante análisis donde el autor relaciona el metro con el contenido en una obra de Píndaro, y determina ciertos criterios de adecuación (contraste de sentido en conexión con diferencia métrica, relaciones entre el baqueo del final del primer período de estrofaantístrofa y el inicial del segundo período de dichos apartados; gliconio final del segundo período de estrofa-antístrofa rodeado de elementos yámbicos, quiasmo de los elementos métricos del último verso del epodo), los mismos que le llevan a pensar que, «aparte de cuestiones estilísticas, ha jugado una baza importante el deseo del

${ }^{27}$ Cf. op. cit., esp. pp. 194-224. Hay que hacer notar, no obstanie, que los *elementos métricos* derivados de su teoría son los que siguen vigentes para la mayoria de los tratadistas, inclusive aquéllos que, como Dain e lrigoin, se inclinan por una libertad de responsión.

28 Cf. ibid., p. 53.

29 Véase de Platón, Rep., 400B. 
poeta de conectar por la métrica y el contenido a Heraclés y sus víctimas ${ }^{30}$ ». Este análisis nos permite apreciar, indudablemente, que la cuestión de la métrica continúa abierta $y$, lo que es más importante, que para el estudioso actual aún se ofrece una multiplicidad de posibilidades para interpretarla en la poesía griega.

\section{Bibliografia}

Daln, A., Leģon sur la métrique grecque. Klincksieck, París, 1944.

Del Grunde, C., "La metrica grecan, Enciclopedia Classica. Soc. Ed. Internazionale, Turín 1960, sec. II, v. I, pp. 133-513.

FESTA, N., Richerche metriche. Saggio di un nuovo metodo per lo studio della metrica greca. Sandron, Roma, 1926.

Gentilt, B., La metrica dei greci. D'Anna, Florencia 1955.

Lenchantin de Gubernatis, M., Manual de prosodia y métrica griega. UNAM, México 1982.

MaAs, P., Greek Metre. Clarendon Press, Oxford 1962.

Macia Aparicio, L., "Adecuación de metro y contenido en la décima Olímpica de Píndaro", Estudios Clásicos, 85, 1980, pp. 33-61.

MARTIN, E., Trois documents de musique grecque. Klincksieck, París 1953.

MARTinez Conesa, J. A., "Glosas al hexámetro homérico", Estudios Clásicos, 64, 1971, pp. 377-89; «Sobre métrica griega», Estudios Clásicos, 64, 1971, Pp. 367-75.

Perusino, F., Il tetrametro giambico catalettico nella commedia greca. Ed. dell'Ateneo, Roma 1968.

Rodriguez Adrados, F., Fernandez-Galiano, M., Gil. L., Lasso de la Vega, J. S., Introducción a Homero. Guadarrama, Madrid 1963.

Ross1, L. E., Metrica e critica stilistica. Ed. deil'Ateneo, Roma 1963.

SÁNCHEZ Ruiperez, M., "Ideas fundamentales sobre métrica griega», Estudios clásicos, I, 1950-52, pp. 239-55.

SNeli, B., Metrica greca. La Nuova Italia, Florencia 1977.

WHITE, J. W., The Verse of Greek Comedy. Macmillan, Londres 1912.

$30 \mathrm{Cf}$, de L. Macta Aparicio, eAdectración de metro y contenido en la décima Olimpica de Píndaron, Estudios Clásicos, 85, 1980, p. 49. 